\title{
Acoustic Waves and Heating Due to Molecular Energy Transfer in an Electric Discharge CO Laser
}

\author{
F. E. C. CULICK, P. I. SHEN, AND W. S. GRIFFIN
}

\begin{abstract}
This paper summarizes analy tical studies and the interpretation of experimental results for the compression and rarefaction waves generated in the cavity of a pulsed CO electric discharge laser. A one-dimensional analysis of acoustic waves is applied to a transversely excited laser. The influences of heating in the cathode fall, heat transfer to the cathode, flow through both the anode and cathode, and bulk heating of the plasma are included. The analysis is used to relate the bulk heating rate to observable features of the pressure and density waves. Data obtained from interferograms and reported elsewhere are used to infer the bulk heating rates in a pulsed CO laser. Results are presented for $\mathrm{CO} / \mathrm{Ar}, \mathrm{CO} / \mathrm{N}_{2}$, and $\mathrm{N}_{2}$ plasmas. Comparison of the data with recent theoretical results for the heating due to electron/ neutral collisions and the anharmonic defect associated with $V-V$ energy transfer shows substantial differences at lower values of total energy deposition. The change of heating with $E / N$ is in fairly good agreement with predicted values.
\end{abstract}

\section{INTRODUCTION}

I N A GAS LASER excited by a pulsed electric discharge, energy is deposited nonuniformly in both space and time. One consequence is that waves of pressure, and therefore density, are excited within the laser cavity. The associated variations in the local index of refraction degrade the optical quality of the output of the device. Formation of these waves may therefore place limits on the maximum pulse length, repetition rate, energy per pulse, and output power obtainable with a specified optical quality.

The presence of waves in the cavity of an electric discharge laser of the sort considered here has been recognized and treated recently elsewhere [1]. In the present work, which was carried out independently of [1], the physical representation of the waves as a problem of acoustics is essentially the same. However, the analytical techniques used are different, and flow through porous electrodes is accounted for. The configuration analyzed is sketched in Fig. 1. Particular emphasis will be placed here on waves generated at the cathode.

As shown in Fig. 1, the optical axis of the laser is normal to the paper. The beam produced by the electron gun ionizes the gas, while a dc sustainer field, applied between the anode and the cathode, provides the electric power required to pump the molecules to vibrationally excited states. It will be assumed here that the sustainer field is constant and applied as a pulse of length $t_{p}$. During the pumping process and for some time subsequently, heating of the gas occurs. For the carbon

Manuscript received January 26, 1976; revised June 1, 1976. This work was supported in part by the Advanced Research Projects Agency of the Department of Defense and monitored by the Office of Naval Research under Contract N0014-72-C-0043.

F. E. C. Culick is with the Department of Engineering, California Institute of Technology, Pasadena, CA 91125.

P. I. Shen and W. S. Griffin are with the Northrop Research and Technology Center, Northrop Corporation, Hawthorne, CA.

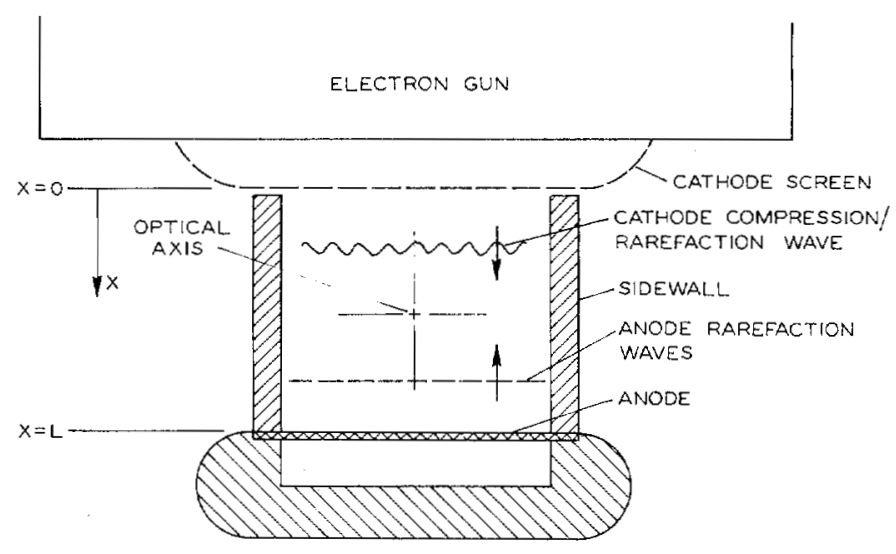

Fig. 1. Sketch of the laser cavity and acoustic waves.

monoxide lasers treated here, there are two important contributions to the heating in most of the cavity: ohmic dissipation associated with elastic collisions between electrons and molecules and the transfer of vibrational energy to translational energy during the process of vibrational-vibrational $(V-V)$ energy transfer. The second arises as a result of the anharmonic defect for the vibrational levels of carbon monoxide [2].

The increase of thermal energy produces an increase of temperature and pressure. Because the electrodes are porous, some of the gas leaks out, causing rarefaction waves to propagate into the cavity. In the thin cathode sheath, the local ohmic heating rate is very high. This produces a relatively high local pressure, generating a steep-fronted compression wave which immediately precedes the rarefaction wave. At the end of the pulse of sustainer voltage, when the intense heating at the cathode ceases, a steep-fronted rarefaction wave is produced. It happens, as shown in Section II, that the rarefaction wave associated with the bulk heating (i.e., that associated with $V-V$ transfer) and the flow through the cathode, has approximately a linear variation in space. Because the heating in the anode sheath is not intense, no sharpfronted waves are produced there.

The features just described are exhibited by the interferograms shown in Fig. 2. These were taken, with a MachZehnder interferometer, for a cavity $1 \mathrm{~m}$ long [3]. In Fig. 3, the fractional change of density is shown, based on the formula (2.27) deduced below. Note that the small number of fringe shifts in the interferograms already suggests the small changes of density depicted explicitly in Fig. 3 .

Because the fluctuations of density, even in the steep portions of the waves, are not large, the problem can reasonably be treated as one of linear acoustics. Solutions for the case of nonuniform and time-varying heat addition are conveniently obtained by the use of a Green's function. This also affords 


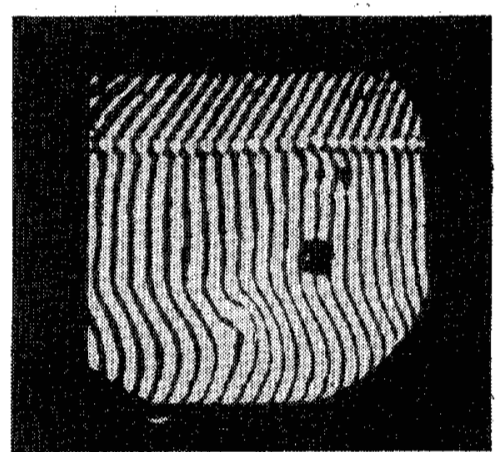

(a)

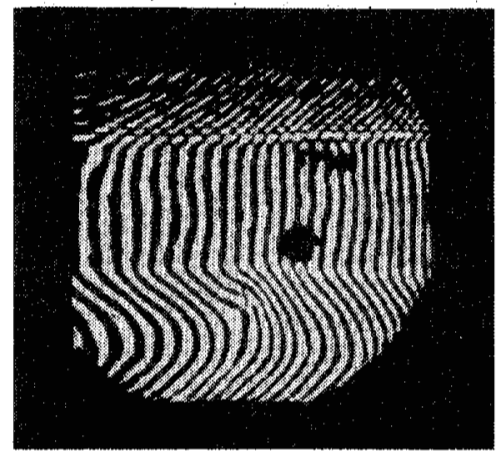

(b)

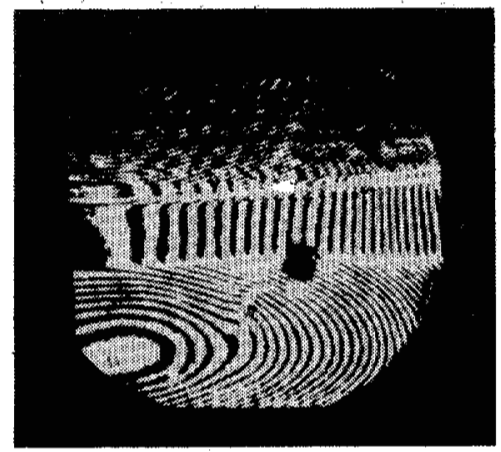

(c)

Fig. 2. Interferograms showing acoustic waves in the laser cavity for different power loadings: (a) $135 \mathrm{~W} / \mathrm{cm}^{3}$, (b) $400 \mathrm{~W} / \mathrm{cm}^{3}$, (c) 1250 $\mathrm{W} / \mathrm{cm}^{3}$. Pictures taken at $180 \mu \mathrm{s}$ after the sustainer pulse.

a simple means of representing the boundary conditions of flow through the electrodes. The formulation given in this work can be used to treat more complicated cases than those treated here.

For simplicity, the heat addition within the cathode sheath is taken to be uniform in a region of thickness $d$. To interpret the interferograms, the limiting case of infinitesimally small $d$, but finite total heat addition in the sheath, is used. So far as the observed waves are concerned, these appear to be good approximations. What this really means is that the details of the processes within the sheath are smeared out or averaged in the process generating the waves.

Owing to the large temperatures which may accompany the heating near the cathode, one might expect that the heat transfer from the gas to the cathode should be substantial. This question is examined in Section III, where it is shown that the influence of the heat loss is confined to a relatively

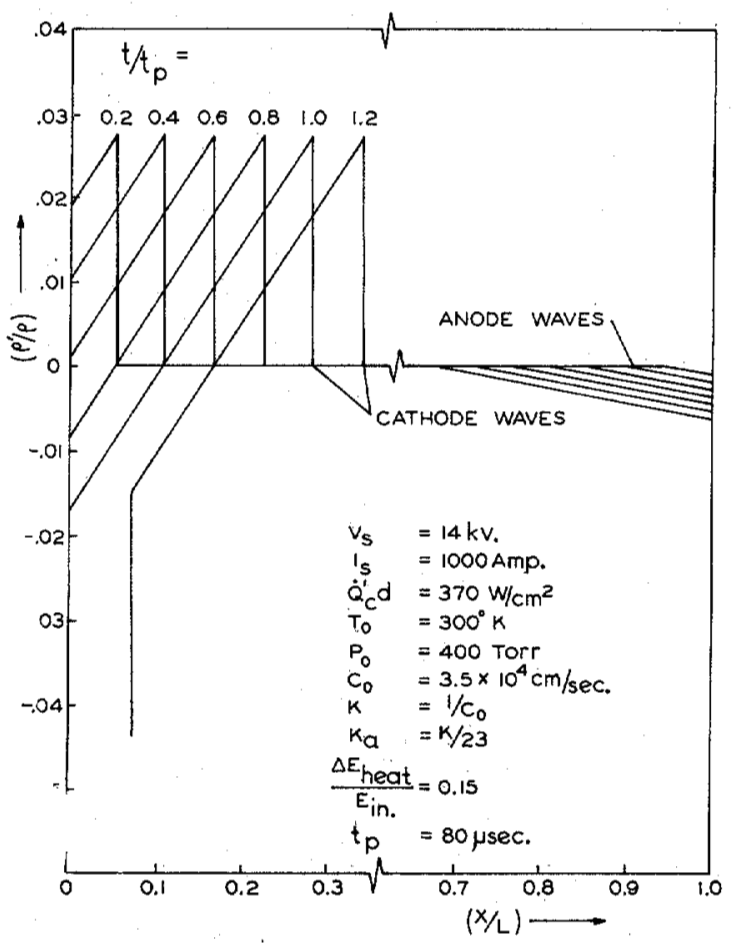

Fig. 3. Calculated density fluctuations according to (2.27). No heat loss to the cathode.

small distance behind the sharp compression wave. It can be ignored for the purposes of Section IV in which the characteristics of the rarefaction wave are used in the following way.

It is a result of the analysis of Section II that the slope of the rarefaction wave (see Fig. 3) is proportional to the bulk heating rate. The slope also depends on the permeability of the cathode, which can be estimated. One can measure the slope on interferograms and can therefore infer the bulk heating rate. The values reported in Section IV confirm the relatively low rates predicted for the $\mathrm{CO}$ laser [2]. However, the theoretical values for the bulk heating are substantially less than those deduced from experiments at lower values of energy deposited in the medium (less than $100 \mathrm{~J} / \mathrm{l}$ ) for an argon/carbon monoxide mixture. The comparison is shown in Fig. 10.

\section{Analysis Without Heat Transfer to THE CATHODE}

\section{A. Formulation}

The experimental evidence discussed above suggests that the waves may be treated as one-dimensional, small-amplitude motions. Because the gas is initially at rest, the average velocity can be taken to be zero. The equation of state is taken to be that for a perfect gas with constant specific heats, so the linearized equations of motion and state are

$$
\begin{aligned}
& \frac{\partial \rho^{\prime}}{\partial t}+\rho_{0} \frac{\partial u^{\prime}}{\partial x}=0 \\
& \rho_{0} \frac{\partial u^{\prime}}{\partial t}+\frac{\partial p^{\prime}}{\partial x}=0 \\
& \rho_{0} \frac{\partial T^{\prime}}{\partial t}+\frac{p_{0}}{\dot{C}_{v}} \frac{\partial u^{\prime}}{\partial x}=\frac{\dot{Q}}{C_{v}} \\
& p^{\prime}=R\left(\rho_{0} T^{\prime}+T_{0} \rho^{\prime}\right) .
\end{aligned}
$$


The heat addition (energy/s · volume) is $\dot{Q}$. From thèse one can form the wave equation for the pressure fluctuations:

$$
\frac{1}{c_{0}^{2}} \frac{\partial^{2} p^{\prime}}{\partial t^{2}}-\frac{\partial^{2} p^{\prime}}{\partial x^{2}}=\frac{\gamma-1}{c_{0}^{2}} \frac{\partial \dot{Q}}{\partial t}
$$

Note that the subscript ( $)_{0}$ denotes quantities for the undisturbed fluid prior to heating, and $c_{0}^{2}=\gamma p_{0} / \rho_{0}$.

Initially, the pressure fluctuation is zero, but is changing at a rate proportional to the heat addition:

$$
\begin{aligned}
p^{\prime} & =0, \quad(t=0, \text { all } x) \\
\frac{\partial p^{\prime}}{\partial t} & =(\gamma-1) \dot{Q} .
\end{aligned}
$$

The second is easily deduced from (2.5) by integrating over a small interval from $t=0-$ to $t=0+$.

Flow through both the cathode and anode must be accounted for. The amount of flow influences the form of the waves generated in the laser cavity itself and also produces waves which propagate in the cavities outside the porous cathode and anode. The consequent changes of pressure within the cathode and anode cavities must subsequently affect the flow through the screens; the laser cavity is obviously acoustically coupled to the cathode and anode cavities. For a more precise treatment of the problem, the pressure fluctuations within the cavities at the cathode and anode should be accounted for. Numerical calculations done as part of this work, but not included here, have shown that the influence of this refinement on the results obtained here is small; for the present it will be ignored. Further remarks on the boundary conditions are given in Section II-F.

Thus the boundary conditions at the electrodes will incorporate the approximation that the pressure is constant in the cavities outside the main laser cavity. The reasonable assumption is made, in addition, that the flow through each electrode is proportional to the pressure difference across the screen. At the cathode, for example, the fluctuation of flow outward is represented by

$$
-\rho_{0} u^{\prime}=K p^{\prime} \text {. }
$$

If $(2.8)$ is differentiated with respect to time, then with (2.2), the boundary condition on $p^{\prime}$ is obtained:

$$
\frac{\partial p^{\prime}}{\partial x}=K \frac{\partial p^{\prime}}{\partial t}, \quad(x=0, \text { all } t)
$$

A similar boundary condition applies at the anode. A complete and proper description of the most general conditions would incorporate that boundary condition from the beginning. However, for most problems of practical interest, one is concerned with the behavior before the waves emanating from the cathode interact with those from the anode (see Figs. 2 and 3). Because the formulation is linear, the waves from the cathode and anode can be treated separately and the results superposed. The second boundary condition may therefore be set on the waves leaving the cathode; that far away the medium is undisturbed:

$$
p^{\prime}=0, \quad(x \rightarrow+\infty, \text { all } t) .
$$

Equation (2.5) with the initial conditions (2.6) and (2.7) and the boundary conditions describe linear waves issuing from the cathode. In the next section, a general solution will be given. This will be evaluated subsequently for the special case of uniform heating in the bulk of the laser cavity, and a thin region of (also uniform) intense heating at the cathode. The waves from the anode will be deduced by a simple transformation.

\section{B. General Solution}

The general solution will be constructed here by first applying the Laplace transform in time. A Green's function will be used to solve the spatial problem. In principle, then, any distribution of the heating rate $\dot{Q}$ can be accommodated. Let a tilde denote the Laplace transform (in the variable $s$ ). Then (2.5), (2.6), and (2.7) can eventually be combined to give

$$
\frac{d^{2} \tilde{p}^{\prime}(x, s)}{d \dot{x}^{2}}-k^{2} \tilde{p}^{\prime}(x, s)=-\frac{\gamma-1}{c_{0}^{2}} s \tilde{\dot{Q}}
$$

where

$$
k=s / c_{0} \text {. }
$$

The transform of the boundary condition (2.9) is

$$
\frac{d}{d x} \tilde{p}^{\prime}(x, s)=s K \tilde{p}^{\prime} \quad(x=0)
$$

and of course the condition (2.10) at infinity gives simply

$$
\tilde{p}^{\prime}(x, s)=0 \quad(x \rightarrow \infty) \text {. }
$$

The Green's function, appropriate to this problem, is governed by the differential equation

$$
\frac{d^{2}}{d x^{2}} G\left(x \mid x_{0}\right)-k^{2} G\left(x \mid x_{0}\right)=\delta\left(x-x_{0}\right)
$$

In addition to satisfying the conditions (2.13) and (2.14), $G\left(x \mid x_{0}\right)$ is subject to the usual requirements that it can be continuous at $x=x_{0}$, but the first derivative has a unit discontinuity. The Green's function is

$$
\begin{aligned}
G_{-}= & \frac{-1}{k(k+s K)}[k \cosh (k x)+s K \sinh (k x)] e^{-k x_{0}} \\
& \cdot\left(x<x_{0}\right) \\
G_{+}= & \frac{-1}{k(k+s K)}\left[k \cosh \left(k x_{0}\right)+s K \sinh \left(k x_{0}\right)\right] e^{-k x} \\
& \cdot\left(x>x_{0}\right) .
\end{aligned}
$$

The solution for the pressure field is

$$
\tilde{p}^{\prime}(x, s)=-\left(\frac{\gamma-1}{c_{0}^{2}}\right) \int_{0}^{\infty} G\left(x \mid x_{0}\right) s \dot{\dot{Q}} d x_{0} .
$$

\section{Waves Generated by the Cathode Fall}

As an approximation to the dissipation of energy in the cathode fall, assume that the heating is uniform and constant in a thin region of thickness $\dot{d}$, for the duration $t_{p}$ of the sustainer pulse: 


$$
\begin{aligned}
\dot{Q}(x, t) & =\dot{Q}_{c} \quad\left(0<x \leqslant d, 0<t \leqslant t_{p}\right) \\
& =\text { otherwise. }
\end{aligned}
$$

Substitution of (2.16) and (2.18) into (2.17) and inversion of the result leads to the formula for the pressure wave gen. erated by the cathode fall:

$$
\frac{p_{f}^{\prime}(x, t)}{(\gamma-1) \dot{Q}_{c}}=f(t, x)-f\left(t-t_{p}, x\right)
$$

where

$$
\begin{aligned}
f(t, x) & =\frac{1}{2}\left(t-\frac{x-d}{c_{0}}\right) U\left(t-\frac{x-d}{c_{0}}\right)-\frac{1-K c_{0}}{2\left(1+K c_{0}\right)} \\
& \left(t-\frac{x+d}{c_{0}}\right) U\left(t-\frac{x+d}{c_{0}}\right)-\frac{K c_{0}}{1+K c_{0}}\left(t-\frac{x}{c_{0}}\right) U \\
& \left(t-\frac{x}{c_{0}}\right)
\end{aligned}
$$

and $U(t-\tau)$ is the unit step function which is zero for $t<\tau$.

The function $f(t, x)$ in (2.19) represents the wave generated when the sustainer pulse is turned on, a relatively sharpfronted compression wave. A rarefaction wave having the same shape is produced when the sustainer pulse is turned off; it is represented by $-f\left(t-t_{p}, x\right)$ in (2.19). At any time $t$, there is a wave of thickness $2 d$ located in the region $c_{0} t-$ $d<x<c_{0} t+d$. It is easy to verify that the function $f(x, t)$ represents the wave sketched in Fig. 4.

If only because the processes in the vicinity of the cathode are necessarily three-dimensional, the thickness $d$ of the cathode region is not known precisely. It is certainly a thin zone, and it is convenient to treat this portion of the wave in the limit $d \rightarrow 0$ but $\dot{Q}_{c}$ finite. The wave therefore becomes a discontinuity, having amplitude proprotional to the heating, $\dot{Q}_{c} d$, per unit area of the cathode:

$$
\begin{aligned}
\operatorname{Lim}_{d \rightarrow 0} p_{f}^{\prime}(x, t)=(\gamma-1) \frac{\dot{Q}_{c} d}{1+K c_{0}} \\
\cdot\left[U\left(t-\frac{x}{c_{0}}\right)-U\left(t-t_{p}-\frac{x}{c_{0}}\right)\right] .
\end{aligned}
$$

This simplified form will be used later in the interpretation of interferograms.

\section{Waves Generated by Bulk Heating}

It is a reasonable approximation that the heating of the plasma is uniform in the volume outside the thin region near the cathode. This is justified also by the observation that the fringes are relatively straight and uniformly spaced in the central areas of the interferograms in Fig. 2. Because the heating causes the pressure to rise, causing gas to flow through the cathode and anode, waves are produced at the electrodes and propagate inward.

For simplicity, the heating is assumed to be both uniform in space and constant in time, even after the pulse is turned off. The transfer of energy from vibration to translation continues as equilibrium is approached subsequent to the pulse. It is

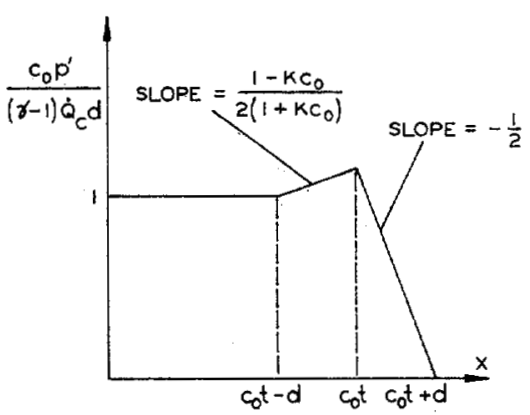

Fig. 4. Sketch of the compression wave produced in the cathode fall when the sustainer pulse is applied.

only for convenience here that $\dot{Q}_{h}$ is assumed to have the same value during and after the pulse. Strictly, $\dot{Q}_{h}$ must tend to zero for $t>t_{p}$. Variations in $\dot{Q}_{h}$ may be one reason for the curvature of the fringes behind the cathode waves shown in Fig. 2. In (2.17) the heat source is represented by

$$
\dot{Q}= \begin{cases}0, & t \leqslant 0 \\ \dot{Q}_{h}, & t>0\end{cases}
$$

with $\dot{Q}_{h}$ constant. Substitution of (2.16) and (2.22) into the formula (2.17) gives

$$
\tilde{p}_{c}^{\prime}(x, s)=(\gamma-1) \dot{Q}_{h} \frac{1}{s^{2}}\left[1-\frac{K c_{0}}{1+K c_{0}} e^{-s x / c_{0}}\right] .
$$

The inverse transform is

$$
\frac{p_{c}^{\prime}(x, t)}{(\gamma-1) Q_{h}}=t-\frac{K c_{0}}{1+K c_{0}}\left(t-\frac{x}{c_{0}}\right) U\left(t-\frac{x}{c_{0}}\right) .
$$

The first term represents the pressure rise which would occur in the cavity if the cathode were impermeable; the second term is the rarefaction wave produced by the flow through the cathode.

A similar result is obtained for the pressure field in the vicinity of the anode, valid before any waves from the cathode cross the cavity. Let $K_{a^{\prime}}$ denote the permeability of the anode, and replace $x$ by $L-x$ to obtain

$$
\frac{p_{a}^{\prime}(x, t)}{(\gamma-1) \dot{Q}_{h}}=t-\frac{K_{a} c_{0}}{1+K_{a} c_{0}}\left(t-\frac{L-x}{c_{0}}\right) U\left(t-\frac{L-x}{c_{0}}\right) .
$$

\section{E. Density Waves in the Laser Cavity}

It is the fluctuations of density in the cavity which are of primary interest, because these are directly related to fluctuations of the index of refraction, which in turn affect the laser beam. The relation between density and pressure is found by using the First Law of Thermodynamics:

$$
C_{v} d T=\frac{d Q}{\rho}+\frac{p}{\rho^{2}} d p
$$

The amount of heat added to any element of gas outside the cathode fall region is simply $\dot{Q}_{h} t$ because $\dot{Q}_{h}$ is constant. Hence, with the temperature change eliminated by use of the equation of state, and with differentials replaced by fluctua- 
tions, one finds

$$
\rho^{\prime}=\frac{p^{\prime}}{c_{0}^{2}}-\frac{\gamma-1}{c_{0}^{2}} \dot{Q}_{h} t
$$

The complete system of pressure waves produced in the cavity is the sum of $(2.20),(2.24)$, and $(2.25) ;(2.26)$ gives

$$
\begin{aligned}
\rho^{\prime}= & (\gamma-1) \frac{\dot{Q}_{c}}{c_{0}^{2}}\left[\frac{1}{2}\left(t-\frac{x-d}{c_{0}}\right) U\left(t-\frac{x-d}{c_{0}}\right)-\frac{1-K c_{0}}{2\left(1+K c_{0}\right)}\right. \\
& \cdot\left(t-\frac{x+d}{c_{0}}\right) U\left(t-\frac{x+d}{c_{0}}\right)-\frac{K c_{0}}{1+K c_{0}} \\
& \left.\cdot\left(t-\frac{x}{c_{0}}\right) U\left(t-\frac{x}{c_{0}}\right)\right] \times\left[1-U\left(t-t_{p}\right)\right] \\
& -(\gamma-1) \frac{\dot{Q}_{h}}{c_{0}^{2}}\left[\frac{K c_{0}}{1+K c_{0}}\left(t-\frac{x}{c_{0}}\right) U\left(t-\frac{x}{c_{0}}\right)\right. \\
& \left.+\frac{K_{a} c_{0}}{1+K_{a} c_{0}}\left(t-\frac{L-x}{c_{0}}\right) U\left(t-\frac{L-x}{c_{0}}\right)\right] .
\end{aligned}
$$

For the limiting case $d \rightarrow 0$,

$$
\begin{aligned}
\rho^{\prime}= & (\gamma-1) \frac{\dot{Q}_{c} d}{c_{0}^{3}\left(1+K c_{0}\right)}\left[U\left(t-\frac{x}{c_{0}}\right)-v\left(t-t_{p}-\frac{x}{c_{0}}\right)\right] \\
& -(\gamma-1) \frac{\dot{Q}_{h}}{c_{0}^{2}}\left[\frac{K c_{0}}{1+K c_{0}}\left(t-\frac{x}{c_{0}}\right) U\left(t-\frac{x}{c_{0}}\right)\right. \\
& \left.+\frac{K_{a} c_{0}}{1+K_{a} c_{0}}\left(t-\frac{L-x}{c_{0}}\right) U\left(t-\frac{L-x}{c_{0}}\right)\right] .
\end{aligned}
$$

Note that the various terms representing the uniform rise of pressure associated with the bulk heating, $\dot{Q}_{h}$, cancel in the formula for the density fluctuations. The approximations used here, in particular that the flows through the cathode and anode are small, imply that the plasma is heated at essentially constant volume.

Fig. 3 is a plot of (2.28) for representative conditions. The difference in slopes of the anode and cathode waves reflects the different permeabilities $\left(K_{a} / K=1 / 23\right)$. The slopes also are proportional to the bulk heating rate, $\dot{Q}_{h}$; examples are shown in Fig. 5. The slope of $K$ used is the limiting value, $1 / c_{0}$, the inverse of the acoustic impedance for the gas. This value is only approximate and valid only for a very porous cathode. If the cathode has appreciable flow resistance, then $K$ would be close to the value for steady-state conditions. No measured values are available. The slope of the wave after the compression front in the cathode, wave is found from (2.28) to be

$$
\left(\frac{\partial \rho_{c}^{\prime}}{\partial x}\right)_{t}=(\gamma-1)\left(\frac{K c_{0}}{1+K c_{0}}\right) \frac{\dot{Q}_{h}}{c_{0}^{3}} .
$$

In Fig. 6, the density profile according to (2.28) is shown for comparison with the interferogram of Fig. 2(b). A heating rate of 15 percent of the input power was assumed. The slopes of the wavefronts and their relative positions in the cavity agree within a few percent. Owing to the poor resolution in the interferogram, the main region of uncertainty is at the steep front of the cathode wave.

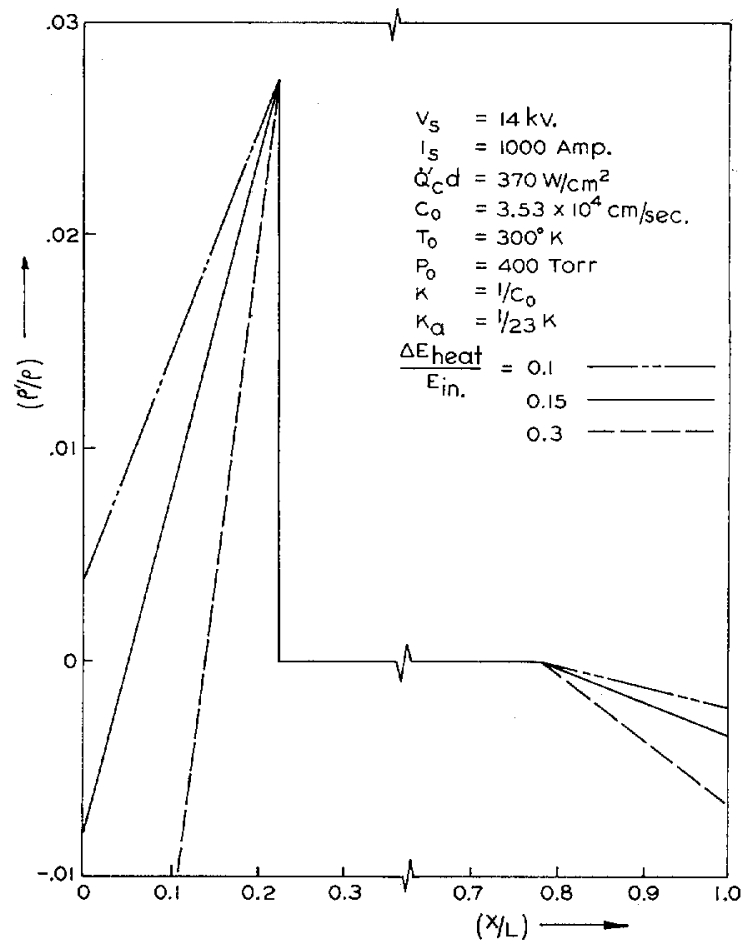

Fig. 5. Illustration of the influence of bulk heating on the cathode and anode waves.

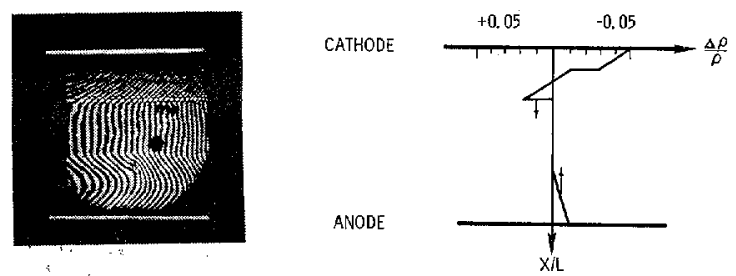

Fig. 6. Comparison of experimental and calculated density variations in a laser cavity.

It should be noted that the distribution of heat addition and the boundary conditions used here are substantially different from those used in [1]. The bulk heating was taken in that work to be nonuniform, and the influence of flow through the electrodes was not examined. If the heat addition is nonuniform in the vicinity of a rigid, impermeable surface, a wave is produced there, even in the absence of flow through the surface. In particular, the anode was apparently solid for the experiments interpreted in [1]; the analysis shows an anode wave because of nonuniform heat addition.

In view of these results, it appears that the simplified analysis constructed here is a farily good approximation for the experimental conditions to be examined here. It will be used in Section IV for inferring values of the bulk heating rate from interferograms. However, an important question arises in connection with the influence of heat transfer to the cathode from the hot gases in the cathode fall. This is examined in Section III.

\section{F. Influence of Pressure Fluctuations in the Electrode Cavities}

As remarked earlier, the expression (2.8) for the boundary condition is an approximation; the influence of pressure 
changes within the cathode cavity and the finite size of the cavity are ignored. More correctly, one should use

$$
-\rho_{0} u^{\prime}=K\left(p^{\prime}-p_{0}^{\prime}\right) \text {, }
$$

where $p_{0}^{\prime}$ is the fluctuation of pressure at the cathode, within the cathode cavity. In order to find $p_{0}^{\prime}$, the problem of wave motions within the cathode cavity must be solved; the solution is then matched to the solution for waves within the main laser cavity.

No essential difficulties are encountered in the solution of this problem by the techniques discussed in Section II-B. The Green's function for this case is

$$
\begin{aligned}
\left(G\left(x \mid x_{0}\right)=\right. & -\frac{1}{k(k+s K)}\left\{[k \cosh (k x)+s K \sinh (k x)] e^{-k x_{0}}\right. \\
& \left.+\frac{(s K)^{2} /(k+s K) \exp -k\left(x_{0}+x\right)}{\tanh \left(k L_{c}\right)+(s K / k+s K)}\right\} \quad\left(x<x_{0}\right) .
\end{aligned}
$$

The formula for $x>x_{0}$ is obtained by using the property $G\left(x \mid x_{0}\right)=G\left(x_{0} \mid x\right)$, which means simply interchanging $x$ and $x_{0} ; L_{c}$ is the depth of the cathode cavity.

The formulas for the pressure waves are now considerably more lengthy than those found for the case $p_{0}^{\prime}=0$ treated above and will not be given here. For the limit $L_{c} \rightarrow 0$, the more general form reduces exactly to the result obtained for $p_{0}^{\prime}=0$. In the limit $L_{c} \rightarrow \infty$, one obtains the result valid before the first reflection from the far side of the cathode cavity returns to the cathode screen. Numerical calculations for that case, with $K=1 / c_{0}$, are practically indistinguishable from those given under the condition $p_{0}^{\prime}=0$. It is for that reason that the simpler results have been used to interpret the observations.

It should be noted that the complete problem accounting for the influences of the boundary conditions at the anode and cathode simultaneously can also be treated. With that solution, one may study the evolution of waves within the laser cavity subsequent to reflections from the anode and cathode. Because the behavior for long times does not appear important for practical applications, it has not been studied in detail.

\section{An Approximation to the Influence of Heat Transfer at the Cathode}

Because of the high heating rates, high temperatures must be expected in the region adjacent to the cathode. Suppose that the voltage drop across the cathode sheath [4] is roughly 370 $\mathrm{V}$ through a distance $d=0.1 \mathrm{~mm}$, and assume that the current density is $1 \mathrm{~A} / \mathrm{cm}^{2}$. For a nitrogen/carbon monoxide gas mixture at 400 torr, the average heating rate in the cathode sheath is $37000 \mathrm{~W} / \mathrm{cm}^{3}$. If the pulse lasts for $t_{p}=100 \mu \mathrm{s}$, the maximum temperature rise is roughly $\dot{Q}_{c} t_{p} / \rho_{0} \dot{C}_{v} \approx 8500 \mathrm{~K}$.

Such high temperatures cannot exist in practice, because heat is rapidly lost to the relatively cool cathode material. It is easy to estimate that if the temperature were so high, the heat transfer exceeds the rate of energy input to the cathode fall. Obviously, the loss of energy to the cathode is significant, and must affect the structure of the compression wave generated in that region.

Qualitatively, one may surmise that the influence of heat transfer is the following. At the beginning of the discharge, when the gas temperature has not changed much from the initial value, there should be little effect. In particular, one might expect that the initial amplitude of the cathode wave should be unaffected. After some time, the gas temperature has risen, heat transfer becomes important, and the compression wave should be weakened. The slope behind the front of the wave would be reduced. But if a steady state for heat conduction is reached, the slope of the compression wave may approach the value determined by the bulk heating rate and the flow through the cathode, (2.29). These ideas will be developed by analyzing the heat transfer as a boundary layer problem at the cathode, and patching the result to the adiabatic solution discussed in Section II.

The linearized energy equation with heat transfer is

$$
\rho_{0} \frac{\partial T^{\prime}}{\partial x}+\frac{p_{0}}{C_{v}} \frac{\partial u^{\prime}}{\partial x}=\frac{\dot{Q}}{C_{v}}+\frac{\lambda}{C_{v}} \frac{\partial^{2} T^{\prime}}{\partial x^{2}},
$$

and the wave equation for the pressure becomes

$$
\frac{\partial^{2} p^{\prime}}{\partial t^{2}}-c_{0}^{2} \frac{\partial^{2} p^{\prime}}{\partial x^{2}}=(\gamma-1) \frac{\partial \dot{Q}}{\partial t}+(\gamma-1) \lambda \frac{\partial}{\partial t}\left(\frac{\partial^{2} T^{\prime}}{\partial x^{2}}\right) \text {. }
$$

Because of the last term in (3.2), this equation is now coupled to both the energy equation and the momentum equation (2.2). The analysis based on the Green's function, used in Section II-B, will not work here. It is for this reason that the heat transfer is treated as a separate boundary layer problem. One assumes that the influence of heat transfer is confined to a region (extending roughly a distance $d$ from the cathode) which is small compared with the characteristic dimension of the adiabatic wave structure. The temperature profile determined in that way is then used in the last term of (3.2) and therefore appears as an additional source of pressure waves.

Two further assumptions are made: 1) the surface temperature of the cathode material does not change appreciably; and 2) the influence of convection can be ignored within the boundary layer. The first may be justified by simple estimates, and is valid because of the high density of the cathode material. The second is made initially to ease the analysis, but the results show that it is valid, principally because the heat transfer rates are much larger than energy transfer by convection within the boundary layer.

The energy equation to be solved in the cathode fall is therefore

$$
\rho_{0} C_{v} \frac{\partial T^{\prime}}{\partial t}=\lambda \frac{\partial^{2} T^{\prime}}{\partial x^{2}}+\dot{Q}
$$

which is now uncoupled from the pressure and velocity fields. Equation (3.3) must be solved subject to the conditions

$$
\begin{array}{ll}
T^{\prime}=0, & x=0, t>0 \\
T^{\prime}=0, & t=0, x>0 \\
T^{\prime}=0, & x \rightarrow \infty, t>0 .
\end{array}
$$




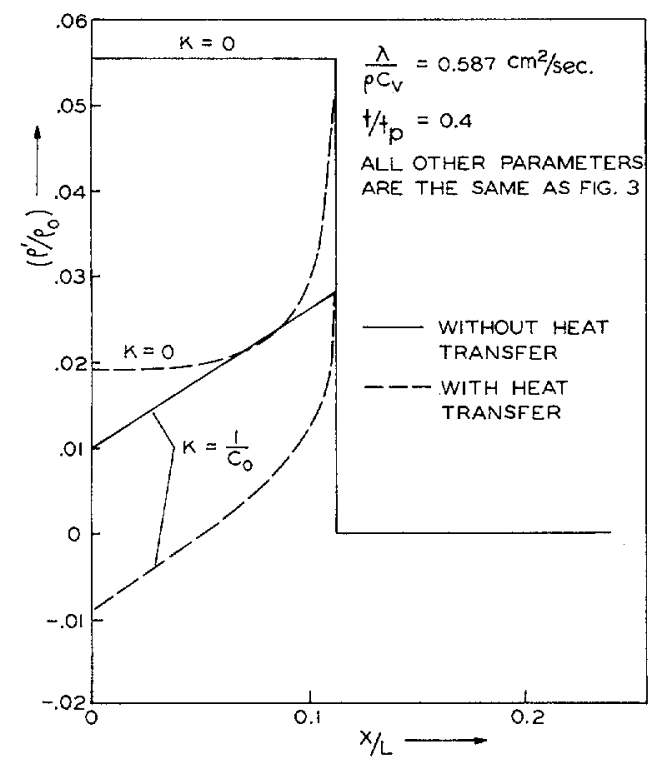

Fig. 7. Illustration of the influence of heat loss to the cathode on the density profile for the cathode wave.

The Laplace transform of (3.3) is

$$
\frac{d^{2} \tilde{T}}{d x^{2}}-\beta^{2} \tilde{T}=-\frac{\dot{Q}}{\lambda}
$$

where

$$
\beta^{2}=\frac{\rho C_{v}}{\lambda} s=\frac{s}{\alpha} \text {. }
$$

The Green's function for (3.5) is

$$
G=-\sqrt{\frac{\alpha}{s}} \begin{cases}\exp \left(-x_{0} \sqrt{\frac{s}{\alpha}}\right) \sinh \left(x \sqrt{\frac{s}{\alpha}}\right), & x<x_{0} \\ \exp \left(-x \sqrt{\frac{s}{\alpha}}\right) \sinh \left(x_{0} \sqrt{\frac{s}{\alpha}}\right), & x>x_{0}\end{cases}
$$

and the temperature profile for uniform heat additions, (2.18), is

$$
\begin{aligned}
\tilde{T}_{-}(x, s)= & \frac{\alpha \dot{Q}_{c}}{\lambda s^{2}}\left[\exp \left(-x \sqrt{\frac{s}{\alpha}}\right)+\frac{1}{2} \exp \left\{\sqrt{\frac{s}{\alpha}}(x-d)\right\}\right. \\
& \left.-\frac{1}{2} \exp \left\{-\sqrt{\frac{s}{\alpha}}(x+d)\right\}-1\right], \quad x<d \\
\tilde{T}_{+}(x, s)= & \frac{\alpha \dot{Q}_{c}}{\lambda s^{2}} \exp \left(-x \sqrt{\frac{s}{\alpha}}\right)\left[\cosh \left(\sqrt{\frac{s}{\alpha}} d\right)-1\right] \\
& x>d
\end{aligned}
$$

Note that the temperature profile decays very rapidly outside the sheath. Equation (3.8) is now used to evaluate the last term of (3.2), and the entire right-hand side appears as the source term in the solution (2.17):

$$
\tilde{p}(x, s)=-\frac{\gamma-1}{c_{0}^{2}} \int_{0}^{\infty}\left[s \tilde{Q}+\lambda s \frac{d^{2} \tilde{T}}{d x_{0}^{2}}\right] G\left(x \mid x_{0} ; s\right) d x_{0} .
$$

For $x>d,(3.9)$ is

$$
\begin{aligned}
-\frac{c_{0}^{2}}{\gamma-1} \tilde{p}(x, s)= & \int_{0}^{d} \dot{Q}_{c} G_{+} d x_{0}+\lambda s \int_{0}^{d} G_{+} \frac{d^{2} \tilde{T}}{d x_{0}^{2}} d x_{0} \\
& +\lambda s \int_{d}^{x} G_{+} \frac{d^{2} \tilde{T}_{+}}{d x_{0}^{2}} d x_{0}+\lambda s \int_{x}^{\infty} G_{-} \\
& \cdot \frac{d^{2} \tilde{T}_{+}}{d x_{0}^{2}} d x_{0} .
\end{aligned}
$$

It is a realistic assumption, which greatly simplifies the result, to take $\alpha s / c_{0}^{2}<<1$. Equation (3.10) then leads to a formula which can be inverted without difficulty. The result can be separated into two parts: the first, arising from the first integral in (3.10), is the adiabatic solution (2.19); the second includes the effects of heat transfer. Denote the latter solution by $p_{h}^{\prime}$; for $t<t_{p}$ this is

$$
\begin{aligned}
& \frac{c_{0}}{(\gamma-1) \sqrt{\alpha} \dot{Q}_{c}} p_{h}^{\prime}(x, t)=\frac{1}{1+K C_{0}}\left[\sqrt{\frac{4}{\pi}\left(t-\frac{x}{c_{0}}\right)}\right. \\
& \cdot \exp \left\{-\frac{d^{2}}{4 \alpha\left(t-x / c_{0}\right)}\right\}-\sqrt{t-\frac{x}{c_{0}}} U\left(t-\frac{x}{c_{0}}\right)-\frac{d}{\sqrt{\alpha}} \\
& \left.\cdot \operatorname{erfc}\left\{\frac{d}{\sqrt{4 \alpha\left(t-x / c_{0}\right)}}\right\}\right]-\frac{\sqrt{\alpha}}{c_{0}}\left[\frac{1}{2} \operatorname{erfc}\left(\frac{x-d}{\sqrt{4 \alpha t}}\right)+\frac{1}{2}\right. \\
& \cdot \operatorname{erfc}\left(\frac{x+d}{\sqrt{4 \alpha t}}-\operatorname{erfc}\left(\frac{x}{\sqrt{4 \alpha t}}\right)-\frac{1}{2} U\left(t-\frac{x-d}{c_{0}}\right)\right. \\
& +\frac{1}{2}\left(\frac{1-K c_{0}}{1+K c_{0}}\right) U\left(t-\frac{x+d}{c_{0}}\right)+\frac{K c_{0}}{1+K c_{0}} U\left(t-\frac{x}{c_{0}}\right)
\end{aligned}
$$

The formula for the fluctuation of density can be deduced as in Section II-E.

Fig. 7 shows the profiles of the density wave for two values of $K$, with and without heat transfer accounted for. Note first, as argued earlier, the initial amplitude of the cathode 


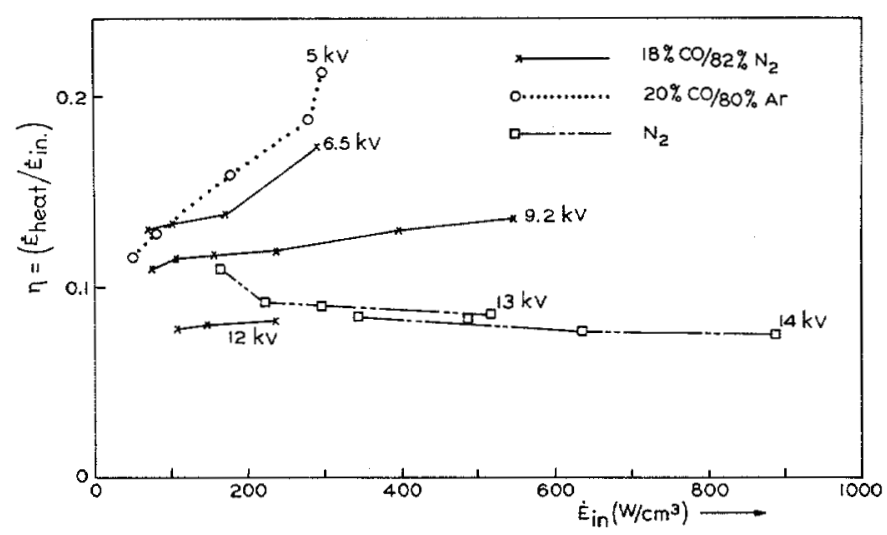

Fig. 8. Experimentally determined bulk heating rates in a 10-1 laser. $p=100$ torr, $T=80 \mathrm{~K}$.

wave is unaffected by heat transfer. As time (or $x / L$ ) increases, an increasing proportion of the energy deposited in the cathode fall is transferred to the cathode itself, and therefore a smaller amount is available to increase the internal energy and pressure of the gas. The pressure is therefore reduced from that produced if the waves were generated with no heat transfer. But a steady state is reached rather quickly $(x / L \sim$ 0.05 ), and at later times a fixed proportion of the energy input. flows to the cathode. The slope of the wave is then the same as that when no heat transfer is accounted for.

The important conclusion of this calculation is that two observable features of the cathode wave-the maximum amplitude and the ultimate slope-can evidently be accurately represented by the result obtained when heat transfer is neglected. A relatively small portion of the wave (roughly 5 percent of the cavity depth for the example shown in Fig. 7) of the wave exhibits the effects of the heat lost to the cathode. These results justify using the simpler analysis of Section II to interpret interferograms of the flow.

\section{Determination of the Bulk Heating Rate}

The formula (2.29) and the corresponding result for the anode wave have been used to infer the bulk heating rate from interferograms. It is convenient to express the bulk heating as a proportion of the total energy input:

$$
\eta=\dot{Q}_{h} / \dot{E}_{\mathrm{in}} \text {. }
$$

Then substitution into (2.29) gives

$$
\eta=\frac{2 \gamma p_{0} c_{0}}{(\gamma-1) \dot{E}_{\text {in }}} \frac{\partial}{\partial x}\left(\frac{\rho^{\prime}}{\rho_{0}}\right)_{\text {cathode }} .
$$

The density gradient is related to the gradient $d N / d x$ of the number of fringes in an interferogram by

$$
\frac{\partial \rho^{\prime}}{\partial x}=\frac{\rho_{s}}{\rho_{0}} \frac{\lambda}{\Omega L_{0}} \frac{d N}{d x}
$$

where $\rho_{s}$ is the gas density at standard conditions, $L_{0}$ is the optical path length, $\Omega$ is the Gladstone-Dale constant [5] and $\lambda$ is the wavelength of the light used.

In [3], details are given for the Mach-Zehnder interferometer assembled to make observations of the density fields in the 10-1 laser described there. Single- and double-pulsed interferograms have been taken for pure $\mathrm{N}_{2}, \mathrm{CO} / \mathrm{Ar}$ mixtures, and

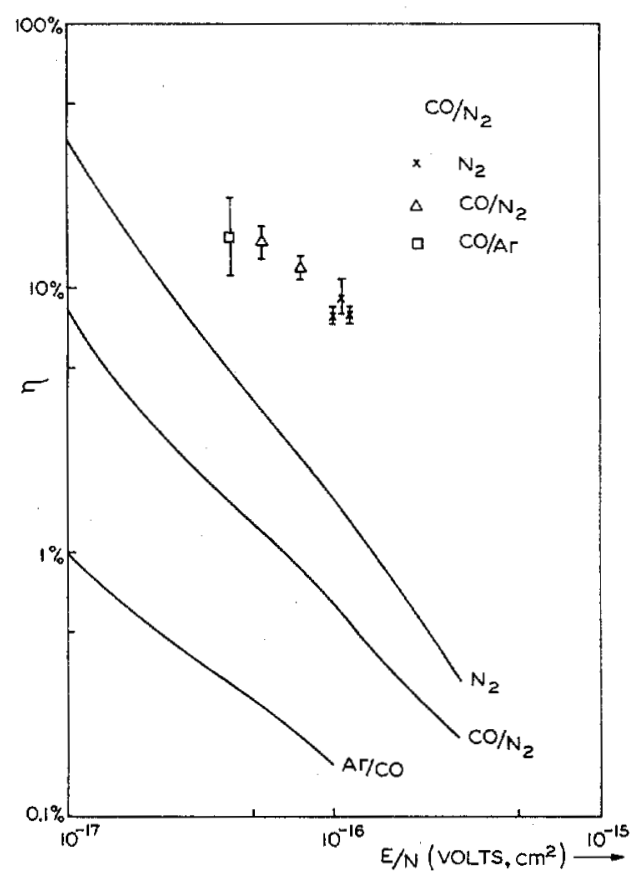

Fig. 9. Comparison of experimentally determined bulk heating rates with the heating calculated for elastic collisions only.

$\mathrm{CO} / \mathrm{N}_{2}$ mixtures. No optical extraction was made during those tests.

Fig. 8 shows the fractional heating rate, deduced from measurement of the fringe gradients and use of (4.3), as a function of input power for three media and several values of sustainer voltage. The data were taken at different times following initiation of the sustainer; it is often more meaningful [6] to use the total energy input, per unit volume, as the independent variable. In Fig. 9, the data are replotted, and the values of applied voltage have been converted to the more fundamental parameter $E / N$, where $N$ is the total number density of neutral particles.

Computed values [7] of the total heating rate due to elastic heating only are compared in Fig. 9 with the data. The results inferred from the interferograms consistently are larger, by roughly a factor of 10 , than the calculated values. This comparison suggests that other sources of heating must be acting; the most obvious is that due to the anharmonic defect in $V-V$ energy transfer, but direct excitation of the rotational 


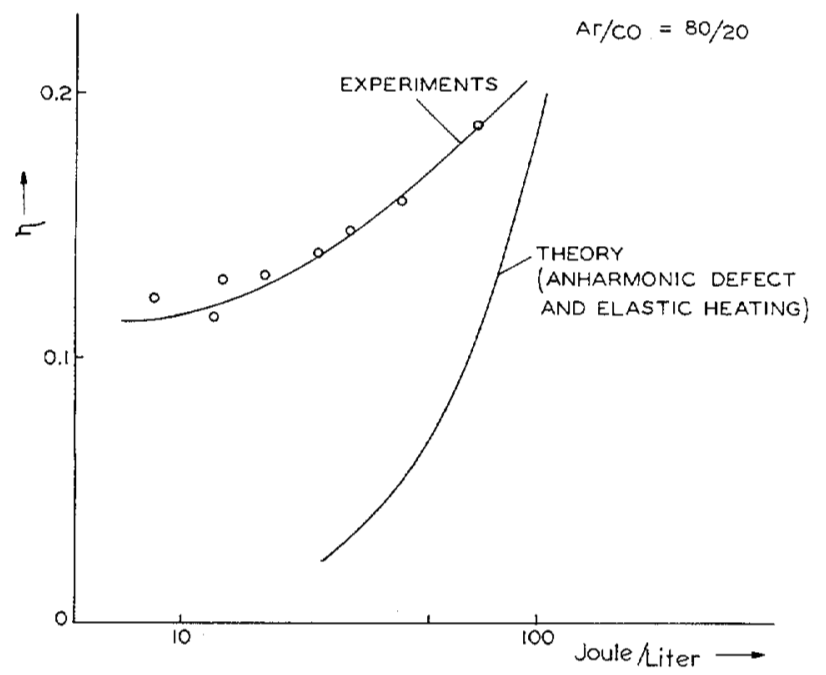

Fig. 10. Comparison of experimentally determined heating rates with the heating calculated for elastic collisions and anharmonic defect (Ar/CO mixture).

degrees of freedom by electron collisions may also contribute. It should be noted, in addition, that within the laser cavity the electric field is strongly nonuniform [7]. The values of $E / N$ may therefore have not been correctly chosen for a valid comparison.

Finally, in Fig. 10, the fractional heating is shown as a function of the total energy deposited for a mixture of argon and carbon monoxide without optical extraction. The calculated values include both elastic heating and heating due to anharmonic defect during $V-V$ energy transfer. Contrary to the predicted behavior, the measured values remain relatively high at the lower values of energy input, although reasonable agreement seems to be found for energies above $100 \mathrm{~J} / 1$. The reasons for the discrepancies are not known. A possible cause [7] is rotational excitation of carbon monoxide, which has not been accounted for in the theoretical work.

\section{ACKNOWLEDGMENT}

The authors thank Dr. W. B. Lacina for informative discussions, and for providing results of his calculations.

\section{REFERENCES}

[1] E. R. Pugh et al., "Optical quality of pulsed electron-beam sustained lasers," Appl. Optics, vol. 13, p. 2512, Nov. 1974.

[2] W. B. Lacina, W. W. Mann, and G. L. McAllister, "Transient oscillator analysis of a high-pressure electrically exicted CO laser," IEEE J. Quantum Electron., vol. QE-9 p. 588, 1973.

[3] G. L. McAllister, R. E. Eguchi, and V. G. Draggoo, "Effects of acoustic waves on the beam quality of high energy CO lasers," Appl. Optics, vol. 14, p. 1290, June 1975.

[4] A. L. Ward, "Calculation of cathode-fall characteristics," J. Appl. Phys, vol. 3, pp. 2789-2794, Sept. 1962.

[5] A. H. Shapiro, The Dynamics and Thermodynamics of Compressible Fluid Flow, vol. I. New York: Ronald Press, 1953, p. 63.

[6] M. M. Mann, "CO lasers-Design considerations," presented at the AIAA 13th Aerospace Sciences Meeting, Pasadena, CA, Jan. 1975, AIAA Paper 75-34.

[7] W. B. Lacina, private communication, to be published.

\title{
Operation-Induced Degradation of GaP Light-Emitting Diodes
}

\author{
AKIRA USAMI AND TOSHIO HAYASHI
}

\begin{abstract}
After GaP red light-emitting diodes (LED's) had been degraded by the forward-biased operation, changes of the light output, the minority carrier lifetime, the photocurrent, and the photocapacitance were measured. There was a linear decreasing relation between the light output and the carrier lifetime. A center which was related to the degradation of the light output was observed by means of photocurrent and photocapacitance measurements. This center's energy level is about $0.8 \mathrm{eV}$ from either the valence or conduction band. This center was observed to increase in concentration with degradation. However, the contribution of this center to the minority carrier lifetime is not established since the capture cross sections of the center are unknown and is not known whether the center occurs on the n-side,
\end{abstract}

Manuscript received February 3, 1976; revised April 26, 1976 and June 9, 1976.

A. Usami is with the Department of Electronics, Nagoya Institute of Technology, Nagoya, Japan.

T. Hay ashi was with the Department of Electronics, Nagoya Institute of Technology, Nagoya, Japan. He is now with the Electrical Communications Laboratory, Nippon Telegraph and Telephone Public Corporation, Tokyo, Japan. or the p-side, or on both sides. This center does not appear to be an oxygen donor.

\section{INTRODUCTION}

M ANY STUDIES have been made of degradation in GaP and GaAs light-emitting diodes (LED's) under forwardbiased operation ([1]- [3]). Degradation is usually caused by an increase in the bulk nonradiative recombination processes and sometimes by surface effects which are neglected in this study. As one of the nonradiative recombination centers, copper in both the GaP LED ([2]) and the GaAs LED ([3]) has been closely related to the device degradation. Two models have been proposed to explain the degradation of GaAs tunnel diodes ([4], [5]). One assumes the migration of interstitial zinc under the forward current stress, and the other assumes the formation of a Frenkel-type defect by the energy released in the nonradiative recombination processes under the 